\title{
Productivity Influence Factors of Tight Sandstone Gas Reservoir with Water Produced
}

\author{
Yongchao Xue $\mathbb{D}$, Qingshuang Jin $(\mathbb{D}$, and Hua Tian \\ State Key Laboratory of Petroleum Resources and Prospecting in China University of Petroleum, Beijing, China \\ Correspondence should be addressed to Yongchao Xue; xyc75@cup.edu.cn
}

Received 16 March 2021; Accepted 16 August 2021; Published 7 September 2021

Academic Editor: Andrew H Manning

Copyright () 2021 Yongchao Xue et al. This is an open access article distributed under the Creative Commons Attribution License, which permits unrestricted use, distribution, and reproduction in any medium, provided the original work is properly cited.

\begin{abstract}
Finding ways to accelerate the effective development of tight sandstone gas reservoirs holds great strategic importance in regard to the improvement of consumption pattern of world energy. The pores and throats of the tight sandstone gas reservoir are small with abundant interstitial materials. Moreover, the mechanism of gas flow is highly complex. This paper is based on the research of a typical tight sandstone gas reservoir in Changqing Oilfield. A strong stress sensitivity in tight sandstone gas reservoir is indicated by the results, and it would be strengthened with the water production; at the same time, a rise to startup pressure gradient would be given by the water producing process. With the increase in driving pressure gradient, the relative permeability of water also increases gradually, while that of gas decreases instead. Following these results, a model of gas-water two-phase flow has been built, keeping stress sensitivity, start-up pressure gradient, and the change of relative permeability in consideration. It is illustrated by the results of calculations that there is a reduction in the duration of plateau production period and the gas recovery factor during this period if the stress sensitivity and start-up pressure gradient are considered. In contrast to the start-up pressure gradient, stress sensitivity holds a greater influence on gas well productivity.
\end{abstract}

\section{Introduction}

The large resource potential and reserve scale of tight sandstone gas reservoirs turn it into a significant strategic succession region regarding newly added proved gas reserves in China $[1,2]$. The pores and throats of tight sandstone gas reservoir are primarily nanoscale and have been distributed complexly, resulting in extremely small tight reservoir permeability along with no natural production generally only in case of being fractured [3-7]. There always exists some associated water in it, due to the particularity of tight sandstone gas reservoir. On the one hand, a massive hydraulic fracturing could enhance the reservoir permeability. On the other hand, it increases the reservoir water saturation because of the invasion of fracturing fluids. The existing water increases the complexity of the flow mechanism in a tight sandstone gas reservoir $[8,9]$ and results in its radical transformation. In addition to the stress sensitivity which is also necessarily considered in single gas flow, the existing water transformed the original single gas flow to gas-water two-phase flow in a tight sandstone gas reservoir $[10,11]$; also, the relative permeability curves undergo changes with variation in the drawdown pressure $[12,13]$. Furthermore, the existing water leads to mutual restriction between water and gas and later also influences the gas flow to be affected by the start-up pressure gradient $[14,15]$. Therefore, water produced in tight sandstone gas reservoir has largely impacted productivity. An in-depth analysis must be given to the effect of water on tight sandstone gas reservoir development in order to decide the primary impacts of productivity of tight sandstone gas reservoir with water produced, thus providing theoretical support for improvement of tight sandstone gas reservoir development.

\section{Characteristic of Gas Flow in Tight Sandstone Reservoirs with Water Produced}

There is a high quartz content in tight sandstone gas reservoirs, and secondary enlargement is common there. (Figure 1(a)). Feldspar is less, but generally, corrosion develops secondary pores (Figure 1(b)). Interstitial materials are also abundant and are mainly mica, which stretch across 


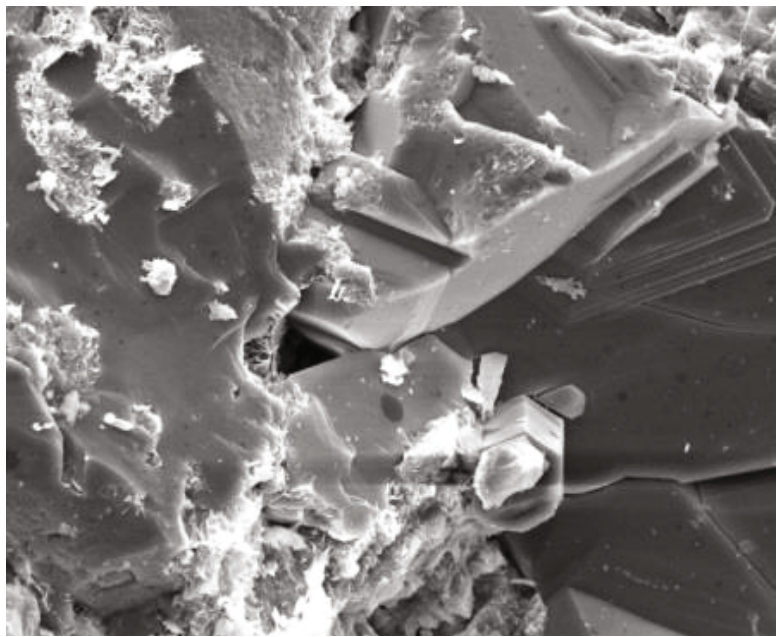

(a) Quartz secondary enlargement

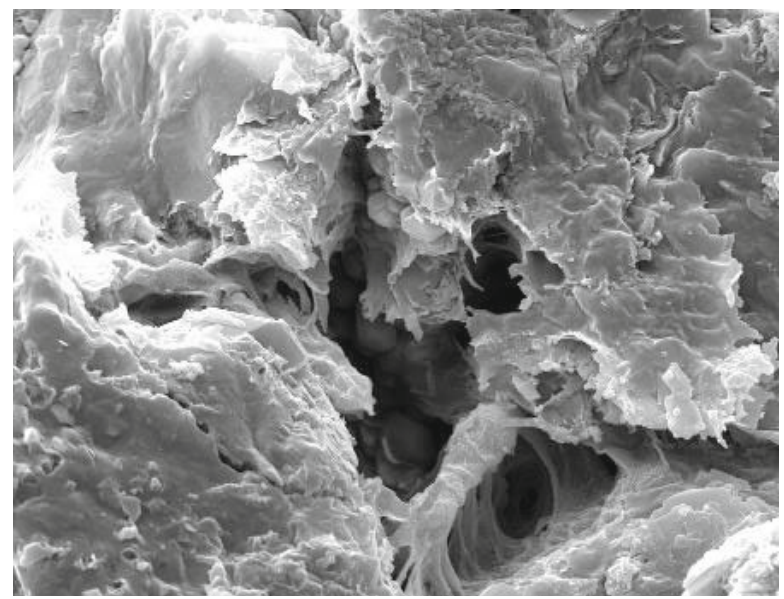

(c) Hydromica on and between granules

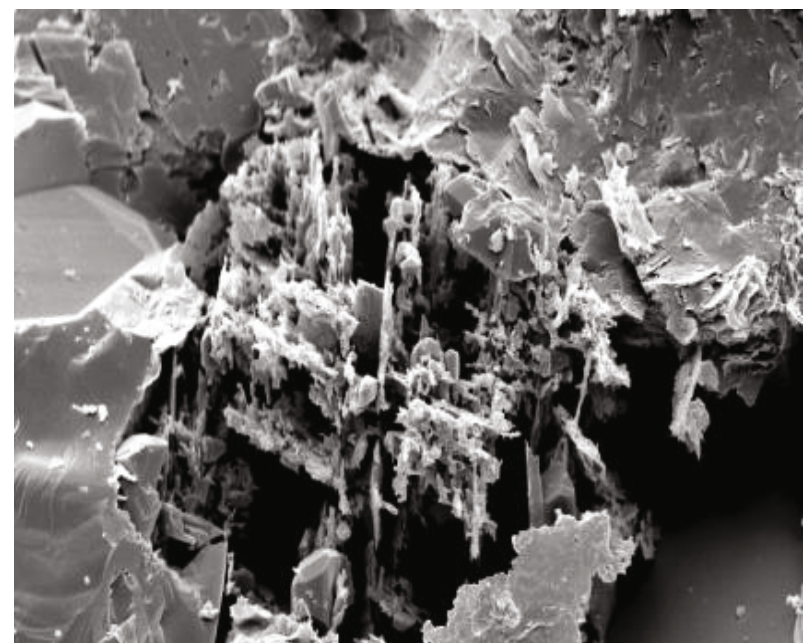

(b) Feldspar corrosion

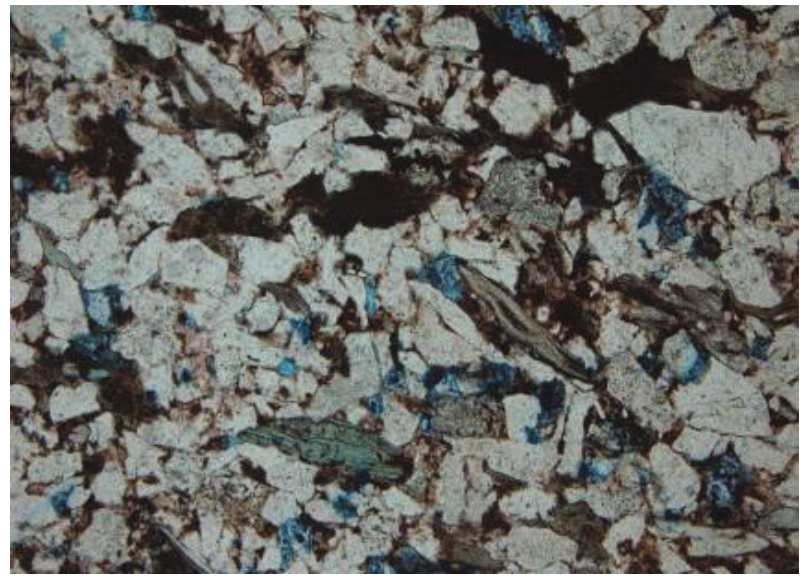

(d) Micropore

FIGURE 1: Tight sandstone gas reservoir percolation medium characteristics.

the pore space such as bridges (Figure 1(c)) and divide the large pores into micropores (Figure $1(\mathrm{~d})$ ). The throat radius of a tight sandstone gas reservoir is approximately $0.5 \mu \mathrm{m}$ primarily, slightly bigger than $4 \mu \mathrm{m}$. More than $40 \%$ of the pore space is controlled by pores whose radius is less than $0.1 \mu \mathrm{m}$. Effective pore space is less than $70 \%$; also, the percentage will be smaller as the stone becomes tighter. It is illustrated by the experiment that the characteristic of gas-water two-phase flow in tight sandstone gas reservoir differs from what is retained by the conventional gas reservoir.

2.1. Characteristic of Gas-Water Two-Phase Flow in Tight Sandstone Gas Reservoir. The existence of water causes the transformation of flow characteristic in tight sandstone gas reservoir, from single phase gas flow to gas-water twophase flow. And due to the small size of pores and throats in tight sandstone gas reservoir, the relative permeability curves are more complicated and manifold. A real reservoir sandstone core of a typical tight sandstone gas reservoir (permeability measured with gas is $0.25 \mathrm{mD}$ ) is used to measure the relative permeability curves with different driving pressure gradients. The experiment result illustrates that the gas-water relative permeability curves with different driving pressure gradients have great difference with one another. As the driving pressure gradient increases, the relative permeability of water increases gradually, while that of gas decreases instead, which indicates that too large drawdown pressure would lead to less gas production rather than more [14] (Figure 2).

According to the above analysis, the relative permeabilities of gas and water are not only the functions of water saturation as conventionally acknowledged but are also related to driving pressure gradient (equation (1)).

$$
\begin{aligned}
& K_{\mathrm{g}}=K(p) K_{\mathrm{rg}}\left(S_{\mathrm{w}}, \nabla p_{\mathrm{g}}\right), \\
& K_{\mathrm{w}}=K(p) K_{\mathrm{rw}}\left(S_{\mathrm{w}}, \nabla p_{\mathrm{w}}\right) .
\end{aligned}
$$

2.2. Effect of Existing Water on Stress Sensibility of Tight Sandstone Gas Reservoir. Generally, it is believed by many researchers that reservoir rock stress sensibility is mainly associated with its absolute permeability and the effective 


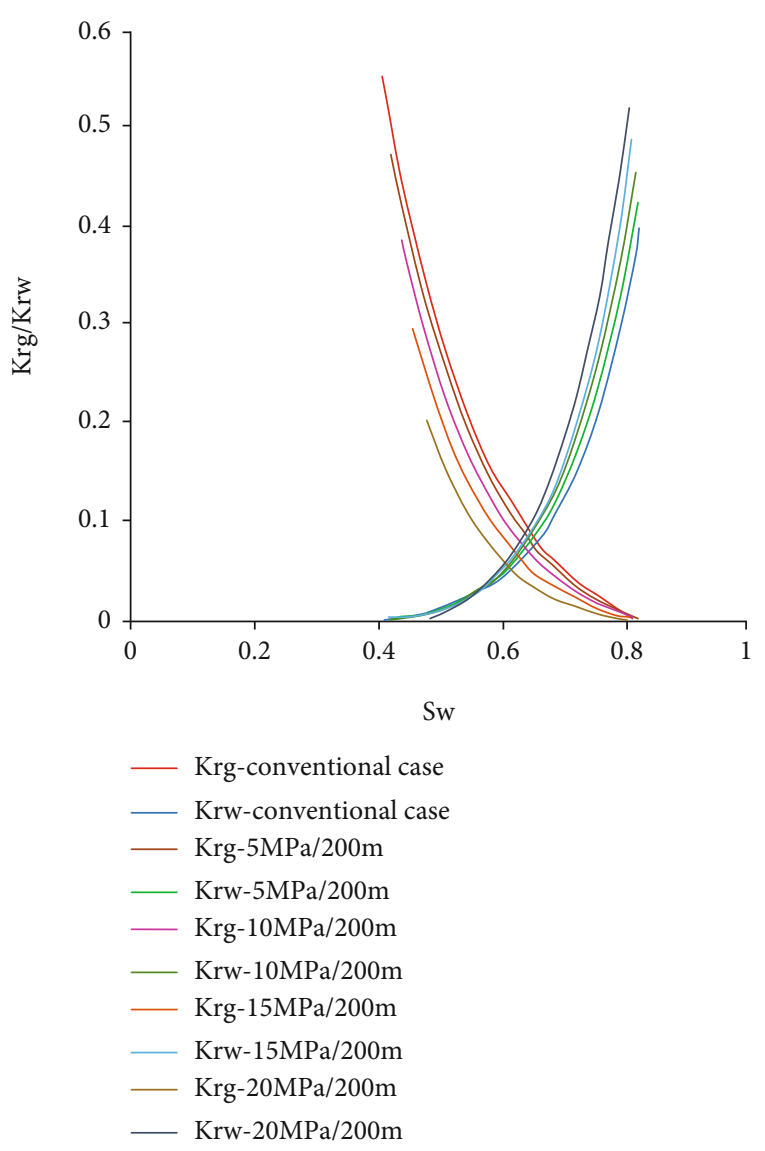

FIGURE 2: Effect of driving pressure gradient on gas-water relative permeability.

stress it bears [16]. In the case of having only a single gas flow in a tight sandstone gas reservoir, the stress sensibility is strong because of the extremely low permeability. Therefore, stress sensitivity has a large impact on productivity of tight sandstone gas reservoirs, while what if water is produced together with gas in tight sandstone gas reservoir (due to high initial water saturation or invading of large volume of fracturing fluid)? Would the water have some impacts on stress sensibility of tight sandstone gas reservoir and further influence gas production? Typical tight sandstone gas reservoir cores (gas log permeability is $0.044 \times 10^{-3} \mu \mathrm{m}^{2}$ ) are selected to measure the influence of different water saturations on the stress sensitivity of the reservoir rock under the same effective stress. Coefficients of tight sandstone gas reservoir stress sensibility with different water saturations are obtained by analysis of the experiment data (Table 1). As shown in Table 1, in other conditions under the condition of invariable, coefficient of tight sandstone gas reservoir stress sensibility increases with water saturation rise. This indicates that the higher the tight sandstone gas reservoir water saturation is, the stronger the stress sensibility will be and the larger impact it will have on gas well productivity. The main reason is that the existing water reduces effective connectivity of gas flow channels and causes mutual restriction of gas and water. For tight sandstone gas reservoir with high initial water saturation, stress change during production would lead to redis- tribution of the water film and then affects gas permeability, which strengthens the gas reservoir stress sensibility and intensifies the corresponding damage. In addition, the physical and chemical interaction between water and tight sandstone gas reservoir minerals reduces compressive strength of the rock, which also enhances the rock stress sensibility. From all of these, we can see that the existence of water does strengthen the tight sandstone gas reservoir stress sensibility. The coefficient of stress sensibility can be calculated by the following formula [17]:

$$
S_{\mathrm{p}}=\frac{1-\left(K / K_{0}\right)^{1 / 3}}{\operatorname{Lg}\left(\sigma / \sigma_{0}\right)} .
$$

From the above analysis, it can be concluded that coefficients of tight sandstone gas reservoir stress sensibility with water produced should be related to water saturation, where $a, b$, and $c$ are undetermined coefficient and can be determined by a correlative experiment of actual oil field.

$$
S_{\mathrm{p}}=\mathrm{a}(K)^{b} e^{c S_{\mathrm{w}}}
$$

2.3. Effect of Existing Water on Start-Up Pressure Gradient. Pascal et al. (in 1981) pointed out that only when the driving pressure gradient is larger than a certain value, the fluid in the porous medium can start to flow. For decades, whether onsite practice or laboratory simulation experiment, start-up pressure gradient is proved to exist extensively, and its theoretical system has been established [18]. When there is only a single gas flow in tight sandstone gas reservoir, start-up pressure gradient would not exist due to the small size of gas molecules, while when water saturation of the tight sandstone gas reservoir is high (water produced), due to the mutual repulsion of gas and water and bigger size of water molecules compared with gas, start-up pressure gradient is generated and then affects the gas well productivity $[19,20]$. Experiment shows that when the water saturation is fixed, there is a power relation between the start-up pressure gradient and the permeability [21]:

$$
\lambda_{\mathrm{g}}=m K^{-n}
$$

\section{Model of Gas-Water Two-Phase Flow in Tight Sandstone Gas Reservoir}

According to the foregoing analysis, the influence of medium deformation, start-up pressure gradient, and effects of driving pressure gradient on gas-water two-phase flow should be considered when establishing a gas-water twophase flow model, and in this way, the flow simulated by the model is closer to the actual flow in the porous medium. Some assumptions are made as follows: the whole process is isothermal and regarded as transient flow; the reservoir is homogeneous and has uniform thickness, and there is only gas-water two-phase flow in it; water is incompressible; gas and water are immiscible; capillary pressure is considered.

The permeability in kinematic equation of tight sandstone gas reservoir is taken as a function of pressure, and 
TABLE 1: Stress sensibility of tight sandstone gas reservoir with different water saturations.

\begin{tabular}{lcccc}
\hline Permeability measured with gas $\left(10^{-3} \mu \mathrm{m}^{2}\right)$ & 0.044 & 0.044 & 0.044 & 0.044 \\
Water saturation (\%) & 0.00 & 31.87 & 46.46 & 56.20 \\
Coefficient of stress sensibility & 0.4768 & 0.8533 & 1.1569 & 1.6426 \\
\hline
\end{tabular}

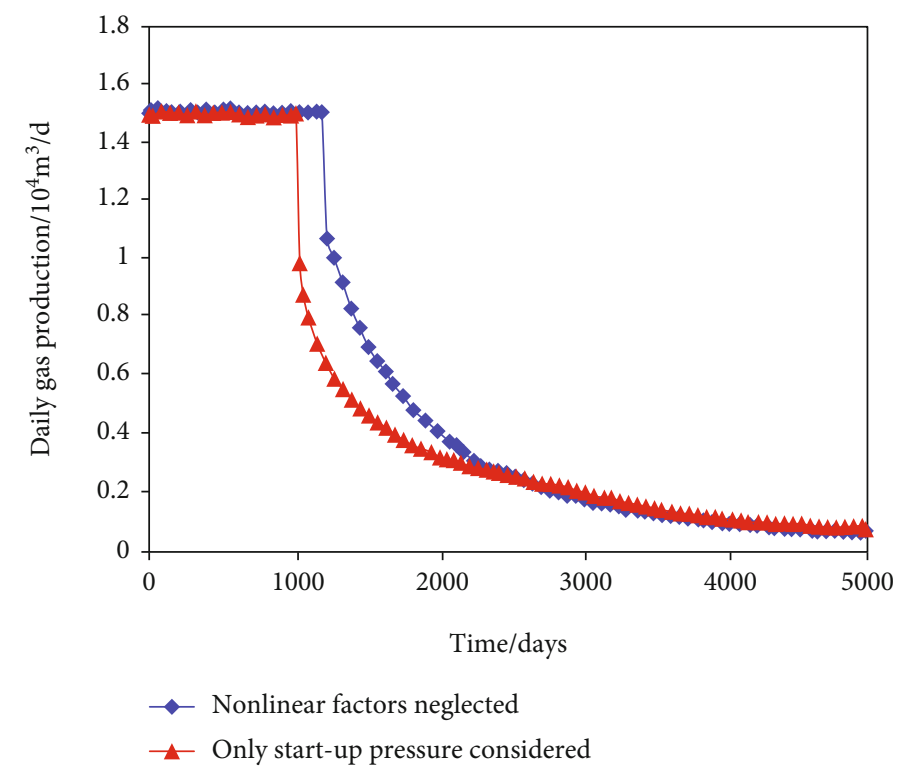

FIgURE 3: Effect of start-up pressure gradient on gas well productivity.

the relative permeability is a function of pressure and water saturation (equations (5) and (6)). Put the kinematic equation and equation of state into equation of continuity, and use the auxiliary equation, boundary conditions, and initial conditions to integrate, and then, establish the basic differential equation of the tight sandstone gas reservoir (equation (7)). Equation (7) is a pressure equation solved by IMPES method. Difference method and numerical calculation module are used to do the computation.

$$
\begin{gathered}
\vec{V}_{\mathrm{g}}=-\frac{k(p) k_{\mathrm{rg}}\left(p, s_{\mathrm{w}}\right)}{\mu_{\mathrm{g}}}\left[\operatorname{grad}\left(p_{\mathrm{g}}\right)-\lambda_{\mathrm{g}}\right] \\
\vec{V}_{\mathrm{W}}=-\frac{k(p) k_{\mathrm{rw}}\left(p, s_{\mathrm{w}}\right)}{\mu_{\mathrm{w}}}\left[\operatorname{grad}\left(p_{\mathrm{w}}\right)-\lambda_{\mathrm{w}}\right], \\
B_{\mathrm{g}}\left\{\nabla \cdot\left[\frac{K(p) K_{\mathrm{rg}}\left(p, s_{\mathrm{w}}\right)}{B_{\mathrm{g}} \mu_{\mathrm{g}}}\left(\nabla p_{\mathrm{g}}-\lambda_{\mathrm{g}}\right)\right]+\frac{q_{\mathrm{g}}}{\rho_{\mathrm{gsc}}}\right\} \\
+B_{\mathrm{W}}\left\{\nabla \cdot\left[\frac{K(p) K_{\mathrm{rw}}\left(p, s_{\mathrm{w}}\right)}{B_{\mathrm{w}} \mu_{\mathrm{w}}}\left(\nabla p_{\mathrm{g}}-\nabla p_{\mathrm{cgw}}-\lambda_{\mathrm{w}}\right)\right]+\frac{q_{\mathrm{w}}}{\rho_{\mathrm{wsc}}}\right\} \\
=\phi C_{\mathrm{t}} \frac{\partial p_{\mathrm{g}}}{\partial t} \cdot
\end{gathered}
$$

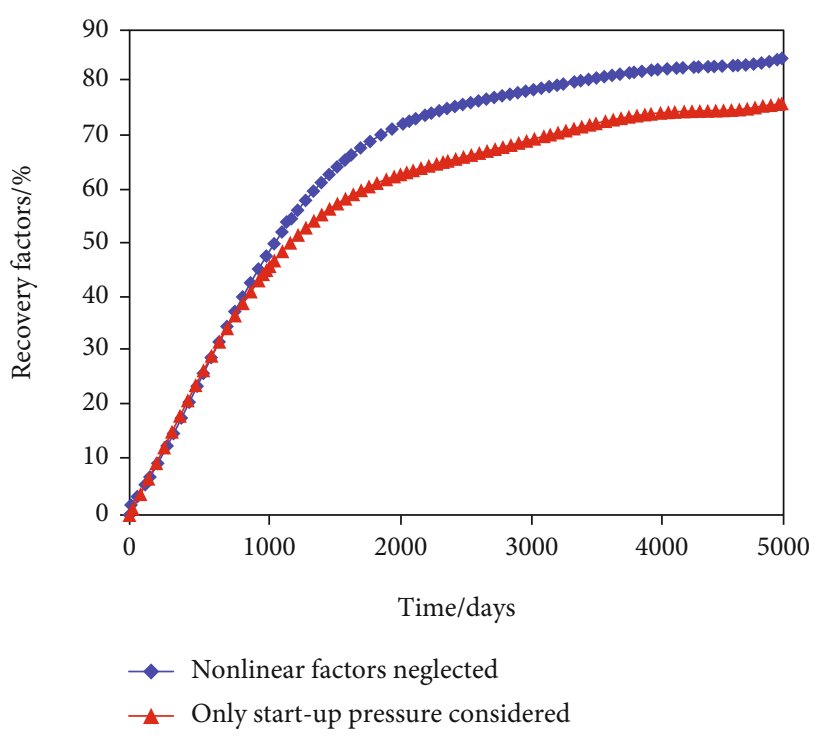

FIGURE 4: Effect of start-up pressure gradient on recovery factor.

\section{Productivity Influence Factors of Tight Sandstone Gas Reservoir}

Take an example of a typical tight sandstone gas reservoir in Changqing Oilfield, and the developed mathematical nonlinear flow model of tight sandstone gas reservoir considering medium deformation, start-up pressure gradient, and 


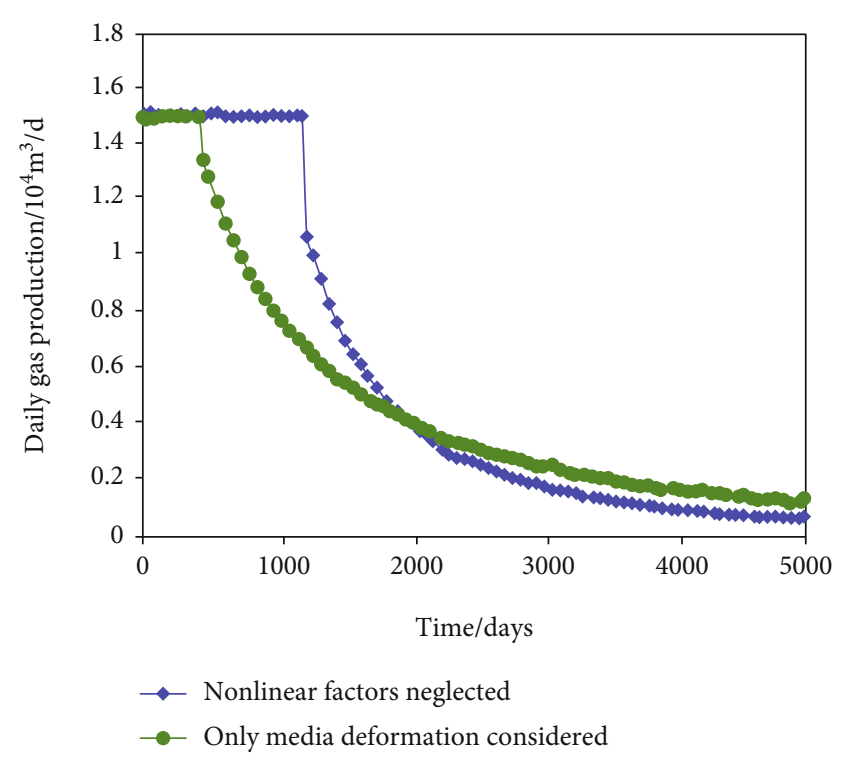

Figure 5: Effect of medium deformation on gas well productivity.

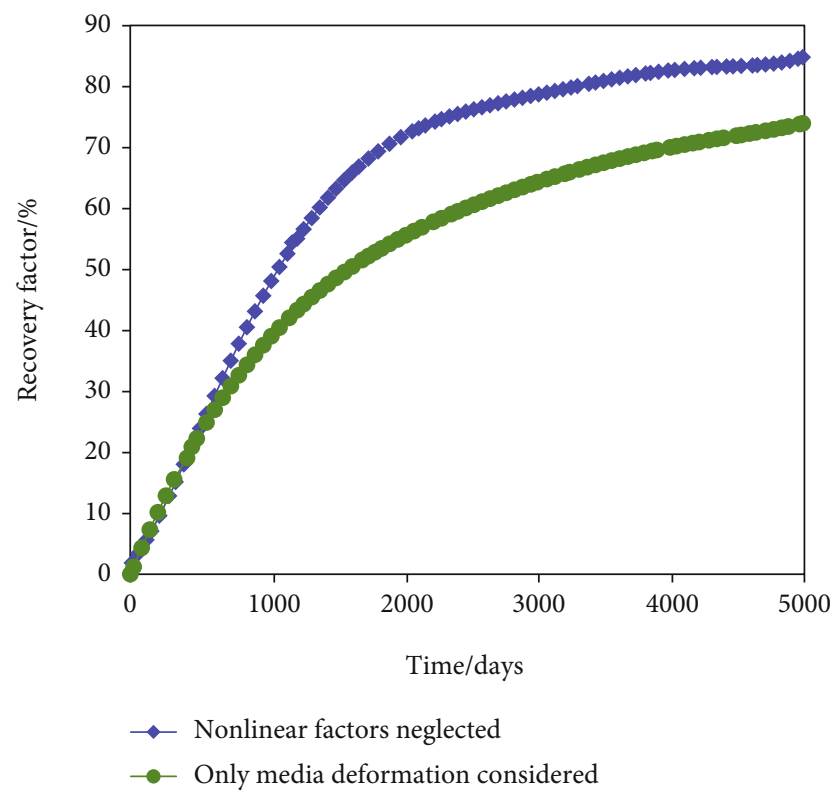

FiguRE 6: Effect of medium deformation on recovery factor.

effects of driving pressure gradient on gas-water two-phase flow is used to calculate and analyze the main productivity influence factors of tight sandstone gas reservoir, thereby providing theoretical support for its development.

The depth of the gas reservoir studied is $3300 \mathrm{~m}$, initial reservoir pressure is $30.5 \mathrm{MPa}$, the average porosity is $8.5 \%$, and average permeability is $0.1 \times 10^{-3} \mu \mathrm{m}^{2}$. The reservoir is developed by vertical hydraulic fracturing well, and the half length of the hydraulic fracture is $100 \mathrm{~m}$, and bottom hole pressure is assumed to be $5 \mathrm{MPa}$. The well is originally set to produce with a constant flow, while when the bottom hole pressure reaches to a certain value, the well will be turned to produce with constant bottom hole pressure. During this

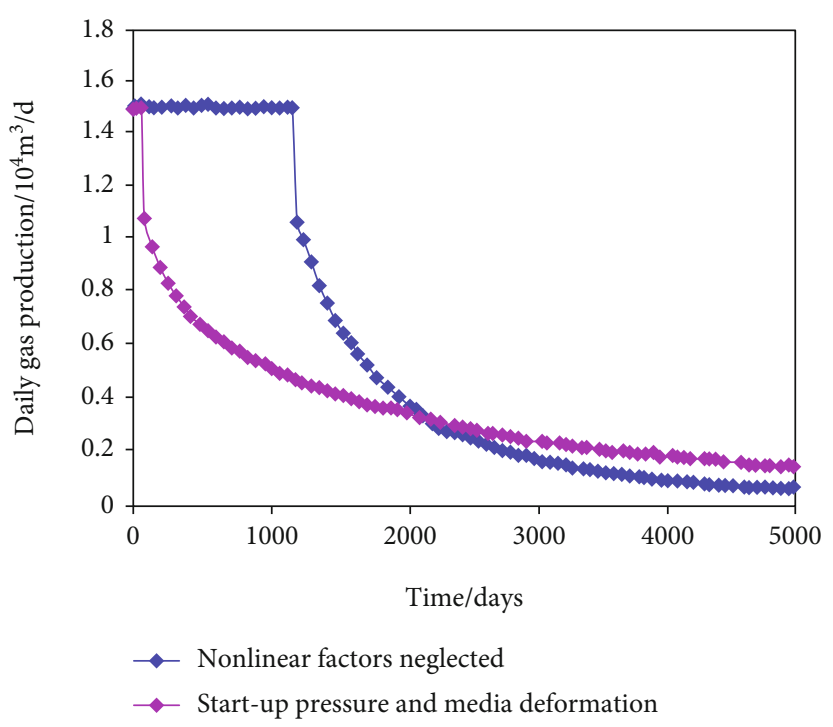

FigURE 7: Effect of nonlinear factors on gas well productivity.

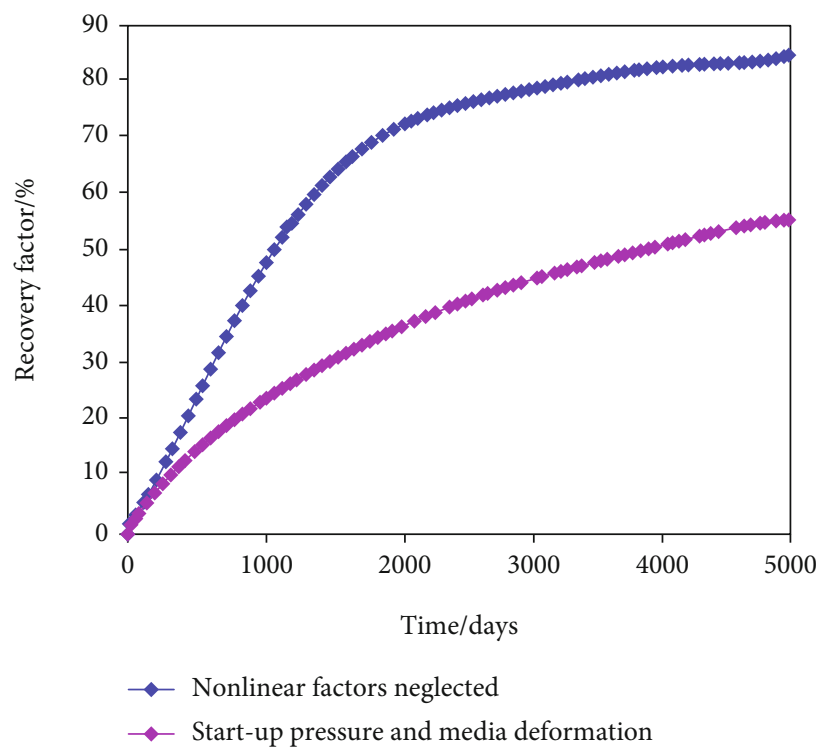

FIGURE 8: Effect of nonlinear factors on recovery factor.

process, different gas-water relative permeability curves are chosen based on the distribution of pressure and driving pressure gradient.

4.1. Effect of Start-Up Pressure Gradient. As previously mentioned, start-up pressure gradient is generated when gas flow in tight sandstone gas reservoir due to the existence of water. To analyze the effect of start-up pressure gradient on gas well productivity, the start-up pressure gradient is considered and disregarded separately in the calculation Figures 3 and 4). Figures 3 and 4 show that when nonlinear factors are neglected, the stable production time is 1201 days and during this period, the recovery percent is $54.39 \%$, and the final recovery ratio is $84.78 \%$, while when start-up 


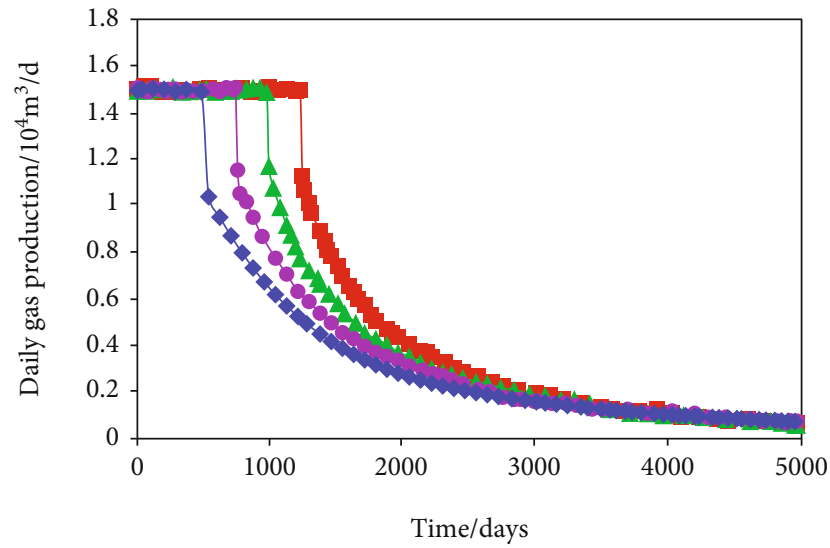

(a) Gas production

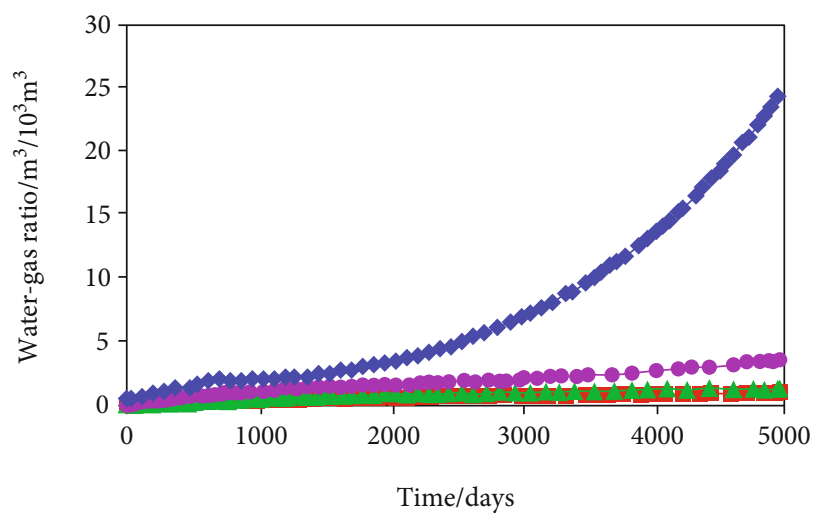

(c) Water-gas ratio

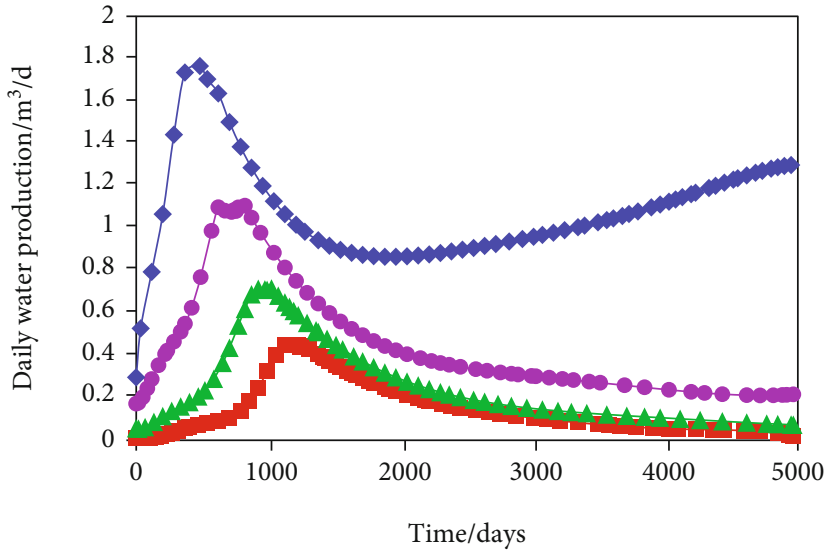

(b) Water production

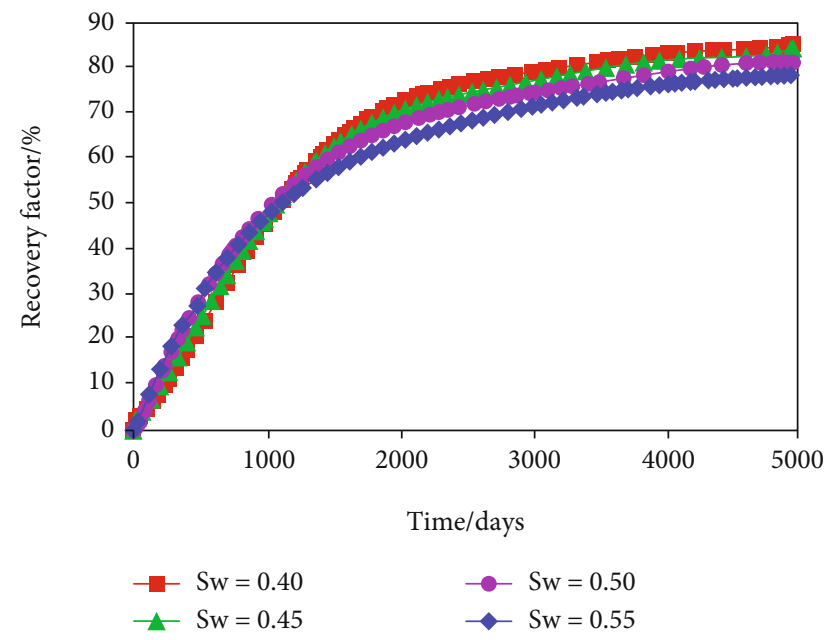

(d) Recovery factor

Figure 9: Gas well productivity with different water saturations.

pressure gradient is taken into account, the stable production time decreases to 1021 days and during this period, the recovery percent is reduced to $44.71 \%$, and the final recovery ratio is $76.45 \%$. In summary, when start-up pressure gradient is taken into account, the time of stable production becomes shorter, and the recovery rate in the plateau period of production and the final recovery coefficient have decreased to a certain extent.

4.2. Effect of Medium Deformation. The existing water intensifies the stress sensibility of tight sandstone gas reservoir and then strengthens the effect of stress sensibility on productivity. Figures 5 and 6 are compared to analyze the effect of stress sensitivity on productivity of tight sandstone gas reservoir. From the figures, we can see that compared with the case when nonlinear factors are neglected, when only stress sensitivity is taken into account, the time of stable production decreases to 421 days, the recovery percent in this period is reduced to $19.06 \%$, and final recovery ratio is $73.93 \%$. In summary, when stress sensitivity is considered, the time of stable production becomes shorter, and both the recovery percent in production plateau period and final recovery factor decrease to some extent.
4.3. Coupling Effect of Start-Up Pressure Gradient and Medium Deformation. When start-up pressure gradient and stress sensitivity have a coupling effect on gas well productivity, as Figures 7 and 8 show, the duration of stable production has a significant decrease from 1201 days to 61 days, the recovery percent in this period decreases to $2.76 \%$, and final recovery ratio is $55.63 \%$. In summary, start-up pressure gradient and stress sensibility mainly affect the stable production period and the recovery percent in this period.

4.4. Effect of Existing Water on Gas Well Productivity. The existing water transforms from the single-phase flow in gas reservoir to gas-water two-phase flow. Figure 9 shows the effect of reservoir water saturation on gas well productivity. When reservoir water saturation increases from 0.40 to 0.55 , the time of stable production decreases from 1225 days to 481 days (Figure 9(a)), water production and water/gas ratio increase dramatically (Figure $9(\mathrm{~b})$ and $9(\mathrm{c})$ ), and the recovery percent decreases by $8.01 \%$ (Figure $9(\mathrm{~d})$ ). In short, higher reservoir water saturation will result in higher water production and significant increase in water/gas ratio. Therefore, drawdown pressure of gas well should be reasonably controlled to reduce the water production. 


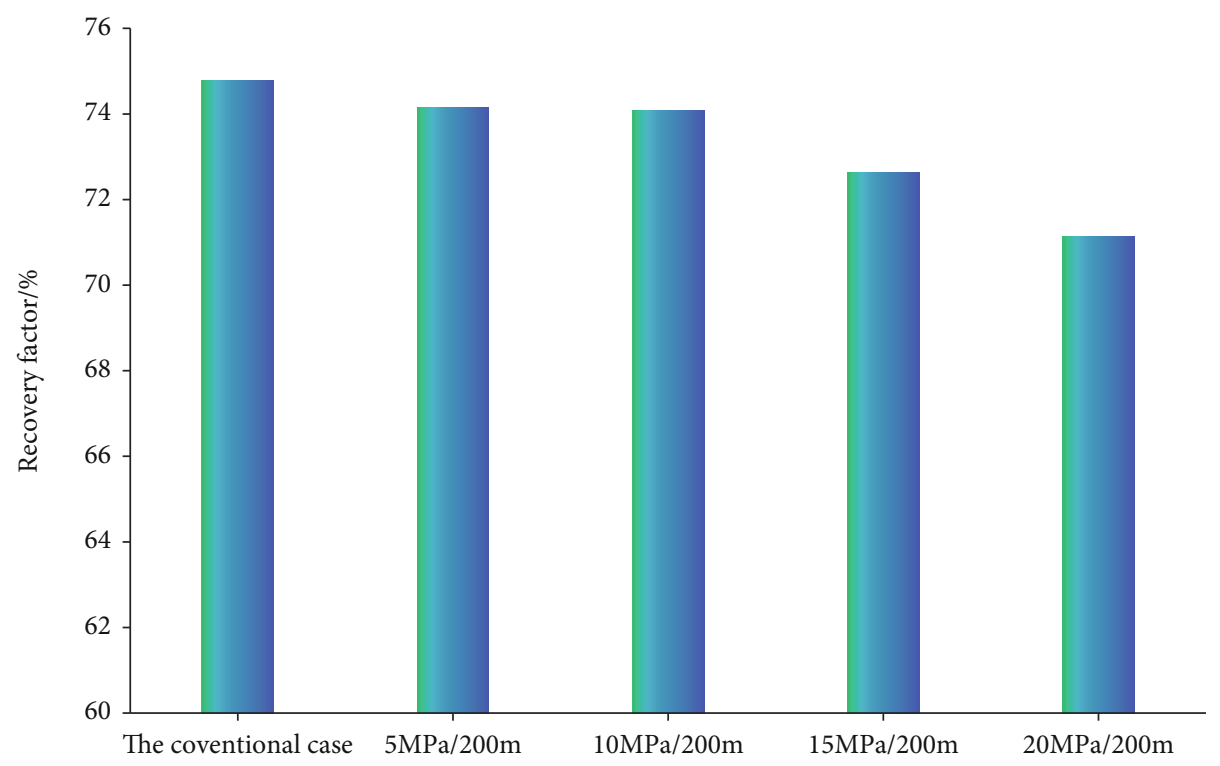

FIGURE 10: Recovery factor with different relative permeability curves.

4.5. Effect of Gas-Water Relative Permeability with Different Driving Pressure Gradients. With the increase of the driving pressure gradient, gas-water relative permeability curves change and affect the mechanism of gas-water two-phase flow. Figure 2 is the collection of relative permeability curves with different driving pressure gradients. Since fluidity of gas is much stronger than water and effected by slippage effect, the relative permeability of gas is greater than that of water under low driving pressure gradients. When the driving pressure gradient increases, water starts to flow in large quantities. The increase of water flow enhances the relative permeability of water, and the gas relative permeability is reduced due to the resistance of the two-phase flow. After the driving pressure gradient increases, more water in the pores becomes movable water and is driven out. At the same time, the water locked by the small throat due to the Jamin effect is also driven out, resulting in a change in the gaswater phase permeability curve.

One set of relative permeability curves (the conventional set) and two sets (the conventional set and the set of curves with a driving pressure gradient of $5 \mathrm{MPa} / 200 \mathrm{~m}$ or $10 \mathrm{MPa} / 200 \mathrm{~m}$ or $15 \mathrm{MPa} / 200 \mathrm{~m}$ or $20 \mathrm{MPa} / 200 \mathrm{~m}$ ) are applied into the calculation model, respectively, and different relative permeability curves will be chosen according to the reservoir pressure. And then, productivities with different driving pressure gradients will be obtained.

From Figure 10, compared with the conventional case, the oil recovery under the corresponding relative permeability of the driving pressure gradients of $5 \mathrm{MPa} / 200 \mathrm{~m}$, $10 \mathrm{MPa} / 200 \mathrm{~m}, 15 \mathrm{MPa} / 200 \mathrm{~m}$, and $20 \mathrm{MPa} / 200 \mathrm{~m}$ decreased by $0.84 \%, 0.94 \%, 2.85 \%$, and $4.87 \%$, respectively. According to engineering error requirements, we can see that one set of permeability curves is sufficient for the calculation if the driving pressure gradient in the reservoir is smaller than $10 \mathrm{MPa} / 200 \mathrm{~m}$, in which case the calculation error can be neglected. However, if the driving pressure gradient in the reservoir is larger than $10 \mathrm{MPa} / 200 \mathrm{~m}$, the relative permeability would have great impact on gas well productivity. Different relative permeability curves should be chosen by the model according to the actual reservoir pressure gradient.

\section{Conclusions}

(1) Pores and throats of the tight sandstone gas reservoir are small, and it has abundant interstitial materials. There is strong stress sensitivity in tight sandstone gas reservoirs, and in the case of water production, it would be strengthened. Simultaneously, the startup pressure gradient would also be aroused by water producing process. The relative permeability of water also increases gradually with the increase in driving pressure gradient; meanwhile, that of gas decreases instead

(2) Considering stress sensitivity, start-up pressure gradient, and the change of relative permeability, a fundamental differential equation of gas-water twophase flow has been built. To analyze the effect of nonlinear factors on productivity, the differential equation is utilized. Result shows that the plateau production period and the gas recovery factor during this period are reduced. If the stress sensitivity and start-up pressure are considered; stress sensitivity holds a greater effect on gas well productivity in contrast to the start-up pressure gradient

(3) Some effect is inflicted upon the gas-water relative permeability by driving pressure gradient. One set of permeability curves is sufficient to perform calculation when the reservoir's driving pressure gradient is smaller than $10 \mathrm{MPa} / 200 \mathrm{~m}$; also, the calculation 
error could be neglected. Nevertheless, when the reservoir's driving pressure gradient exceeds more than $10 \mathrm{MPa} / 200 \mathrm{~m}$, the relative permeability immensely impacted the gas well productivity, in which case the model must select different relative permeability curves according to the actual reservoir pressure gradient.

\section{Nomenclature} $k_{j}$

$k_{r j}$ :

$k$ :

$S_{j}$ :

$\nabla p_{j}$ :

$S_{p}:$

$\lambda_{j}:$

$\vec{v}_{j}:$

$\mu_{j}$ :

$B_{\mathrm{g}}, B_{\mathrm{w}}:$

$\rho_{\mathrm{gsc}}, \rho_{\mathrm{wsc}}$ :

$\nabla p_{\text {cgw }}:$

$q_{j}:$

$\phi:$

$C_{\mathrm{t}}$ :

$p_{j}:$

$S_{\mathrm{p}}$ :

$\sigma_{0}$ :

$K_{0}$ :

$\sigma:$

$K$ :

$a, b, c$ :

$m$ :

$n:$

$d_{1}, d_{2}, d_{3}:$

Permeability of single phase, $\mu \mathrm{m}^{2}$

Relative permeability of single phase

Absolute permeability, $\mu \mathrm{m}^{2}$

Fluid saturation, decimal

Driving pressure drop, $\mathrm{MPa} / \mathrm{m}$

Coefficient of tight sandstone reservoir stress sensibility

Start-up pressure gradient, $\mathrm{MPa} / \mathrm{m}$

Velocity of flow in porous media, $\mathrm{cm} / \mathrm{s}$

Fluid viscosity, $\mathrm{mPa} \cdot \mathrm{s}$

Volume factors of gas and water, decimal

Densities of gas and water in standard

condition, $\mathrm{g} / \mathrm{cm}^{3}$

Capillary pressure of gas and water,

$\mathrm{MPa} / \mathrm{m}$

Mass flow rate of fluid $j, \mathrm{~g} / \mathrm{s}$

Porosity, decimal

Comprehensive compression factor, $\mathrm{MPa}^{-1}$

Pressure of $j$ phase

Coefficient of stress sensitivity, decimal

Initial stress value, $\mathrm{MPa}$

Initial permeability, $10^{-3} \mu \mathrm{m}^{2}$

Effective stress, $\mathrm{MPa}$

Permeability measured with gas,

$10^{-3} \mu \mathrm{m}^{2}$

Parameters to be calculated

Coefficient $m$ has a power relation with

$S_{\mathrm{w}}, m=e^{d_{1} S_{\mathrm{w}}}$

Coefficient $n$ has a linear relation with $S_{\mathrm{w}}$ , $n=d_{2} \cdot S_{\mathrm{w}}+d_{3}$

Undetermined coefficient and can be determined by correlative experiment of actual oil field.

\section{Data Availability}

The data used to support the findings of this study are available from the corresponding author upon request.

\section{Conflicts of Interest}

The authors declare that they have no conflicts of interest.

\section{Acknowledgments}

The authors gratefully acknowledge the support by the Strategic Cooperation Technology Projects of China National Petroleum Corporation (CNPC) and China University of Petroleum, Beijing (CUPB) (No. ZLZX2020-02-04).

\section{References}

[1] C. Jia, M. Zheng, and Y. Zhang, "Unconventional hydrocarbon resources in china and the prospect of exploration and development," Petroleum Exploration and Development, vol. 39, no. 2, pp. 129-136, 2012.

[2] K. Zhang, "Natural gas supply-demand situation and prospect in China," Natural Gas Industry, vol. 34, no. 1, pp. 10-17, 2014.

[3] P. Jia, M. Ma, C. Cao, L. Cheng, H. Yin, and Z. Li, "Capturing dynamic behavior of propped and unpropped fractures during flowback and early-time production of shale gas wells using a novel flow-geomechanics coupled model," Journal of Petroleum Science and Engineering, vol. 2021, article 109412, 2021.

[4] P. Jia, D. Wu, H. Yin, Z. Li, L. Cheng, and X. Ke, "A Practical Solution Model for Transient Pressure Behavior of Multistage Fractured Horizontal Wells with Finite Conductivity in Tight Oil Reservoirs," Geofluids, vol. 2021, Article ID 9948505, 10 pages, 2021.

[5] X. Ma, A. Jia, and J. Tan, "Tight sand gas development technologies and practices in China," Petroleum Exploration and Development, vol. 39, no. 5, pp. 572-579, 2012.

[6] R. K. Zhu, B. Bai, J. Cui et al., "Research advance of microstructure in unconventional tight oil and gas reservoirs," Journal of Palaeogeography, vol. 15, no. 5, pp. 615-623, 2013.

[7] Y. Kang and P. Luo, "Current status and prospect of key techniques for exploration and production of tight sandstone gas reservoirs in China," Petroleum Exploration and Development, vol. 34, no. 2, pp. 239-245, 2007.

[8] Q. Lan, H. Dehghanpour, J. Wood, and H. Sanei, "Wettability of the Montney tight gas formation," SPE Reservoir Evaluation \& Engineering, vol. 18, no. 3, pp. 417-431, 2014.

[9] X. Zheng, C. Zhigang, and L. Weichuan, "Gas/water flowing ability influence experimental study of permeability stress sensibility in tight gas reservoir," Well Logging Technology, vol. 37, no. 4, pp. 360-363, 2013.

[10] C. Cao, L. Cheng, X. Zhang, and J. Shi, "Numerical simulation investigation on well performance integrated stress sensitivity and Sand production," Geofluids, vol. 2021, pp. 1-11, 2021.

[11] C. Cao, L. Cheng, X. Zhang, P. Jia, and J. Shi, "Seepage proxy model and production forecast method based on multivariate and small sample," Chinese Journal of Theoretical and Applied Mechanics, vol. 53, no. 8, pp. 1-10, 2021.

[12] H. Yong, L. Xizhe, W. Yujin et al., "Physical simulation on gas percolation in tight sandstone," Petroleum Exploration and Development, vol. 40, no. 5, pp. 580-584, 2013.

[13] J. Fang, P. Guo, X. Xiao et al., "Gas-water relative permeabilities measurement of high temperature and high pressure tight gas reservoirs," Petroleum Exploration and Development, vol. 41, no. 6, pp. 1-4, 2014.

[14] S. S. Gao, L. Y. Ye, W. Xiong et al., "Seepage mechanism and strategy for development of large and low permeability and tight sandstone gas reservoirs with water content," Journal of Oil and Gas Technology, vol. 35, no. 7, pp. 93-99, 2013. 
[15] F. Hao, L. S. Cheng, O. Hassan, J. Hou, C. Z. Liu, and J. D. Feng, "Threshold pressure gradient in ultra-low permeability reservoirs," Petroleum Science and Technology, vol. 26, no. 9, pp. 1024-1035, 2008.

[16] P. Dong, G. Lei, B. Y. Ji, and S. B. Tian, "Nonlinear seepage regularity of tight sandstone reservoirs with consideration of medium deformation," Chinese Journal of Rock Mechanics and Engineering, vol. 32, Supplement 2, pp. 3187-3196, 2013.

[17] L. Lan, Y. Kang, Y. Chen et al., "Discussion on experimental methods and evaluation indexes of reservoir stress sensitivity evaluation," Drilling Fluid \& Completion Fluid, vol. 22, no. 3, pp. 1-4, 2005.

[18] F. Pascal, H. Pascal, and D. W. Murray, "Consolidation with threshold gradient, Inter J for Numerical and Analytical Mehtods in Geomechanics," Consolidation with threshold gradients., vol. 52, no. 5, pp. 247-261, 1981.

[19] M. L. Brown, E. Ozkan, R. S. Raghavan, and H. Kazemi, "Practical solutions for pressure transient responses of fractured horizontal wells in unconventional reservoir," in SPE125043-MS presented at the SPE Annual Technical conference and exhibition, 4-7 October, New Orleans: SPE, 2009.

[20] J. G. Osorio, H.-Y. Chen, and W. Lawrence, "Numerical simulation of coupled fuid-flow/geomechanical behavior of tight gas reservoirs with stress sensitive permeability," in SPE39055-MS Presented at the Latin American and Caribbean Petroleum Engineering Conference, 30 August-3 September, Rio de Janeiro, Brazil: SPE, 1997.

[21] Z. Yang, X. Li, S. Liu, S. S. Gao, L. Y. Ye, and J. Liu, “Threshold pressure effect of low permeability tight gas reservoir in Sulige," Acta Petrolei Sinica, vol. 36, no. 3, pp. 347-354, 2015. 